\title{
A functional model associated with a generalized Nevanlinna pair
}

\author{
EVGEN NeIMAN
}

(Presented by M. M. Malamud)

\begin{abstract}
Let $\mathcal{L}$ be a Hilbert space and let $\mathcal{H}$ be a Pontryagin space. For every selfadjoint linear relation $\widetilde{A}$ in $\mathcal{H} \oplus \mathcal{L}$ the pair $\{I+\lambda \psi(\lambda), \psi(\lambda)\}$, where $\psi(\lambda)$ is the compressed resolvent of $\widetilde{A}$, is a normalized generalized Nevanlinna pair. Conversely, every normalized generalized Nevanlinna pair is shown to be associated with some selfadjoint linear relation $\widetilde{A}$ in the above sense. A functional model for this selfadjoint linear relation $\widetilde{A}$ is constructed.
\end{abstract}

2010 MSC. 47B38, 47B25, 47B32, 47B50.

Key words and phrases. selfadjoint relation, reproducing kernel Pontryagin space, generalized Nevanlinna pair, generalized Fourier transform.

\section{Introduction}

In 1946 M. G. Krein introduced in [12] the notion of the $Q$-function of a symmetric operator $A$ in a Hilbert space with finite deficiency indices $(m, m)$, which plays an important role in the description of generalized resolvents of $A$. Later on M. G. Krein and H. Langer in [14] have generalized this notion to the case of a symmetric operator $A$ with infinite indices acting in a Pontryagin space. In that paper it was shown that the $Q$-function uniquely determines a simple symmetric operator $A$ up to unitary equivalence. Moreover, in [14] a functional model for a symmetric operator relied on $\varepsilon$-construction was introduced and investigated. This 
model has allowed them to solve an inverse problem for the $Q$-function, that is to find a criterion for a generalized Nevanlinna operator valued function to be the $Q$-function of a $\pi$-Hermitian operator.

Functional models for symmetric operators in Hilbert spaces in terms of the $Q$-function were constructed in $[1,15]$. Different functional models for symmetric operators have been used by V. A. Derkach and M. M. Malamud in [10] (see also [16]) to solve the inverse problem for the Weyl function. Namely, starting with a uniformly strict $R$-function $M(\cdot)$ (i.e. $R$-function satisfying $0 \in \rho(\operatorname{Im} M(i)))$ the authors in [10] constructed a model symmetric operator $A$ and a boundary triplet for $A^{*}$ such that the corresponding Weyl function coincides with $M(\cdot)$. This result has also been extended to a wider class of strict $R$-functions (that is $R$-functions with $\operatorname{ker} \operatorname{Im} M(i)=\{0\})$ in order to realize any such $R$ function as the Weyl function corresponding to a generalized boundary triplet (see [10]).

Later on a concept a generalized boundary triplet was generalized in [7] where a notion of a boundary relation and the corresponding Weyl family was introduced. Using this notion the authors of [7] have realized arbitrary Nevanlinna pair $\{\varphi, \psi\}$ as the Weyl family of some symmetric operator corresponding to a boundary relation (a realization theorem). The proof in [7] was based on the Naimark dilation theorem and the so called main transform. Later on another proof of the realization theorem from [7] have been presented in $[4,5]$ where more general models for symmetric operators were introduced.

In the present paper given a generalized Nevanlinna pair $\{\varphi, \psi\}$ we construct a functional model for a selfadjoint linear relation $\widetilde{A}$ in Pontryagin space such that $\varphi, \psi$ are recovered from $\widetilde{A}$ via (2.4). To make the paper clear for a wide audience we follow the scheme of [6] and use the notion of the selfadjoint linear relation $\widetilde{A}$ rather then the notion of the boundary relation $\Gamma$. In fact, one can treat $\widetilde{A}$ as the main transform of a boundary relation $\Gamma$ and then the main result can be reformulated in terms of $\Gamma$.

The paper is organized as follows. In Section 1 definitions of $N_{\kappa}$-pairs and normalized $N_{\kappa}$-pairs are given. In Section 2 we consider a pair $\{\varphi, \psi\}$ generated by a selfadjoint relation $\widetilde{A}$ in a Pontryagin space and show that it is a normalized $N_{\kappa}$-pair. In Theorem 3.1 we prove the converse result. Moreover, a functional model for the selfadjoint relation $\widetilde{A}$ is constructed. In the rest of the paper properties of a generalized Fourier transform, associated with this model are studied. We also proved the unitary equivalence of an arbitrary $\mathcal{L}$-minimal selfadjoint linear relation $\widetilde{A}$ to the model relation $A(\varphi, \psi)$ in the reproducing kernel Pontryagin space. 


\section{Generalized Nevanlinna pairs}

Let $\mathcal{L}$ be a Hilbert space. By a kernel is meant a function $\mathrm{K}_{\omega}(\lambda)$ on $\Omega \times \Omega$ with values in the space of continuous operators on a Hilbert space $\mathcal{L}(\Omega \subset \mathbb{C})$. We say that the kernel $\mathrm{K}_{\omega}(\lambda)$ has $\kappa$ negative squares and write $s q_{-} \mathrm{K}=\kappa$ if for any choice set of points $\omega_{1}, \ldots, \omega_{n}$ in $\Omega$, vectors $u_{1}, \ldots, u_{n}$ in $\mathcal{L}$ and $\xi_{j}$ in space $\mathbb{C}^{n}$ the quadratic form

$$
\sum_{i, j=1}^{n}\left(\mathrm{~K}_{\omega_{j}}\left(\omega_{i}\right) u_{j}, u_{i}\right)_{\mathcal{L}} \xi_{j} \bar{\xi}_{i}
$$

has at most $\kappa$ negative eigenvalues, and for some choice of $n, \omega_{j}, u_{j}$ such matrix has exactly $\kappa$ negative squares ([2]).

Definition 1.1. A pair $\{\Phi, \Psi\}$ of $[\mathcal{L}]$-valued functions $\Phi(\cdot), \Psi(\cdot)$ meromorphic on $\mathbb{C} \backslash \mathbb{R}$ with a common domain of homomorphy $\mathfrak{h}_{\Phi \Psi}$ is said to be a $N_{\kappa}$-pair (a generalized Nevanlinna pair) if:

(i) the kernel

$$
\mathrm{N}_{\omega}^{\Phi \Psi}(\lambda)=\frac{\Psi(\bar{\lambda})^{*} \Phi(\bar{\omega})-\Phi(\bar{\lambda})^{*} \Psi(\bar{\omega})}{\lambda-\bar{\omega}},
$$

has $\kappa$ negative square on $\mathfrak{h}_{\Phi \Psi}$;

(ii) $\Psi(\bar{\lambda})^{*} \Phi(\lambda)-\Phi(\bar{\lambda})^{*} \Psi(\lambda)=0$ for all $\lambda \in \mathfrak{h}_{\Phi \Psi}$;

(iii) for all $\lambda \in \mathfrak{h}_{\Phi \Psi} \cap \mathbb{C}_{+}$there is $\mu \in \mathbb{C}_{+}$such that

$$
0 \in \rho(\Phi(\lambda)-\mu \Psi(\lambda)) \text { and } 0 \in \rho(\Phi(\bar{\lambda})-\bar{\mu} \Psi(\bar{\lambda})) .
$$

Two $N_{\kappa}$-pairs $\{\Phi, \Psi\}$ and $\left\{\Phi_{1}, \Psi_{1}\right\}$ are said to be equivalent, if $\Phi_{1}(\lambda)=\Phi(\lambda) \chi(\lambda)$ and $\Psi_{1}(\lambda)=\Psi(\lambda) \chi(\lambda)$ for some operator function $\chi(\cdot) \in[\mathcal{H}]$, which is holomorphic and invertible on $\mathfrak{h}_{\Phi \Psi}$. The set of all equivalence classes of $N_{\kappa}$-pairs in $\mathcal{L}$ will be denoted by $\widetilde{N}_{\kappa}(\mathcal{L})$. We will write, for short, $\{\Phi, \Psi\} \in \widetilde{N}_{\kappa}(\mathcal{L})$ for the generalized Nevanlinna pair $\{\Phi, \Psi\}$.

If $\Phi(\lambda) \equiv I_{\mathcal{L}}$ where $I_{\mathcal{L}}$ is the identity operator in the space $\mathcal{L}$ then the Definition 1.1 means that $\Psi(\lambda)$ is an $N_{\kappa}(\mathcal{L})$-function in the sense of [13]. Recall that the class $N_{\kappa}(\mathcal{L})$ consists of meromorphic in $\mathbb{C}_{+} \cup \mathbb{C}_{-}$operator valued functions $\Psi(\lambda)$ such that $\Psi(\bar{\lambda})=\Psi(\lambda)^{*}$, and the kernel

$$
\mathrm{N}_{\omega}^{\Psi}(\lambda)=\frac{\Psi(\lambda)-\Psi(\omega)^{*}}{\lambda-\bar{\omega}}
$$

has $\kappa$ negative squares on $\mathfrak{h}_{\Psi}$ - the domain of holomorphic $\Psi$. In this case the condition (iii) is satisfied automatically. Clearly, if $\{\Phi, \Psi\}$ is $N_{\kappa}$-pair such that $0 \in \rho(\Phi(\lambda)) \lambda \in \mathfrak{h}_{\Phi \Psi}$, then it is equivalent to the pair $\left\{I_{\mathcal{L}}, \Psi(\lambda) \Phi(\lambda)^{-1}\right\}$, where $\Psi \Phi^{-1} \in N_{\kappa}(\mathcal{L})$. 
Definition 1.2. An $N_{\kappa}$-pair $\{\phi, \psi\}$ is said to be a normalized $N_{\kappa}$-pair if:

(iii') $\varphi(\lambda)-\lambda \psi(\lambda) \equiv I_{\mathcal{L}}$ for all $\lambda \in \mathfrak{h}_{\varphi \psi}$.

Clearly, every $N_{\kappa}$-pair $\{\Phi, \Psi\}$ such that $0 \in \rho(\Phi(\lambda)-\lambda \Psi(\lambda))$ for $\lambda \in \mathfrak{h}_{\Phi \Psi}$ is equivalent to a unique normalized $N_{\kappa}$-pair $\{\varphi, \psi\}$ given by

$$
\varphi(\lambda)=\Phi(\lambda)(\Phi(\lambda)-\lambda \Psi(\lambda))^{-1}, \quad \psi(\lambda)=\Psi(\lambda)(\Phi(\lambda)-\lambda \Psi(\lambda))^{-1} .
$$

\section{2. $N_{\kappa}$-pair corresponding to a selfadjoint linear relation and a scale}

Let $\mathfrak{H}$ be a vector space with a Hermitian form $[\cdot, \cdot]_{\mathfrak{H}}: \mathfrak{H} \times \mathfrak{H} \rightarrow \mathbb{C}$. Two elements $u$ and $v$ of $\mathfrak{H}$ are said to be orthogonal if $[u, v]_{\mathfrak{H}}=0$. Similarly, two subspaces of $\mathfrak{H}$ are said to be orthogonal if every element of the first is orthogonal to every element of the second. The linear space $(\mathfrak{H},[\cdot, \cdot])$ is called a Pontryagin space if there exists a direct orthogonal decomposition $\mathfrak{H}=\mathfrak{H}_{+} \oplus \mathfrak{H}_{-}$, where $\mathfrak{H}_{+}$with the form $[\cdot, \cdot]_{\mathfrak{H}}$ is a Hilbert space and $\mathfrak{H}_{-}$with the form $-[\cdot, \cdot]_{\mathfrak{H}}$ is a Hilbert space of finite dimension. The space $\mathfrak{H}$ is called Pontryagin space with $\kappa$ negative squares $\left(\Pi_{\kappa}\right.$-space) if the dimension of $\mathfrak{H}_{-}$is $\kappa<\infty$ ([2]).

We will use the notion of a linear relation in a space $\mathfrak{H}$. Recall, that a subspace $T$ of $\mathfrak{H}^{2}$ is called the linear relation in $\mathfrak{H}$. For a linear relation $T$ in $\mathfrak{H}$ the symbols $\operatorname{dom} T, \operatorname{ker} T, \operatorname{ran} T$, and mul $T$ stand for the domain, kernel, range, and the multivalued part, respectively. The adjoint $T^{+}$is the closed linear relation in $\mathfrak{H}$ defined by (see [2])

$$
T^{+}=\left\{\{h, k\} \in \mathfrak{H}^{2}:[k, f]_{\mathfrak{H}}=[h, g]_{\mathfrak{H}},\{f, g\} \in T\right\} .
$$

Recall that a linear relation $T$ in $\mathfrak{H}$ is called symmetric (selfadjoint) if $T \subset T^{+}\left(T=T^{+}\right.$, respectively).

Let $\mathcal{H}$ be a Pontryagin space and $\mathcal{L}$ be a Hilbert space.

Definition 2.1. A linear relation $\widetilde{A}=\widetilde{A}^{*}$ in $\mathcal{H} \oplus \mathcal{L}$ is said to be $\mathcal{L}$ minimal if

$$
\mathcal{H}_{0}:=\overline{\operatorname{span}}\left\{P_{\mathcal{H}}(\widetilde{A}-\lambda)^{-1} \mathcal{L}: \lambda \in \rho(\widetilde{A})\right\}=\mathcal{H},
$$

where $P_{\mathcal{H}}$ is the orthogonal projection onto the Pontryagin space $\mathcal{H}$.

Let $\widetilde{A}$ be a selfadjoint linear relation in $\mathcal{H} \oplus \mathcal{L}$ and let $P_{\mathcal{L}}$ be the orthogonal projection onto the scale space $\mathcal{L}$. Define the operator valued functions

$\varphi(\lambda):=I_{\mathcal{L}}+\lambda P_{\mathcal{L}}(\widetilde{A}-\lambda)^{-1} \uparrow_{\mathcal{L}}, \quad \psi(\lambda):=P_{\mathcal{L}}(\widetilde{A}-\lambda)^{-1} \uparrow_{\mathcal{L}} \quad(\lambda \in \rho(\widetilde{A}))$. 
Clearly,

$$
\varphi(\lambda)^{*}=\varphi(\bar{\lambda}), \quad \psi(\lambda)^{*}=\psi(\bar{\lambda}) \quad(\lambda \in \rho(\widetilde{A})) .
$$

Proposition 2.1. Let $\mathcal{H}$ be a $\Pi_{\kappa}$-space, let $\mathcal{L}$ be a Hilbert space and let $\widetilde{A}$ be a selfadjoint linear relation in $\mathcal{H} \oplus \mathcal{L}$. The pair of operator valued functions $\{\varphi, \psi\}$ associated with $\widetilde{A}$ via $(2.4)$ is a normalized $N_{\kappa^{\prime}}$-pair where $0 \leq \kappa^{\prime} \leq \kappa$. If, additionally, the linear relation $\widetilde{A}$ is $\mathcal{L}$-minimal then $\kappa^{\prime}=\kappa$.

Proof. In view of the properties (2.5) the kernel $\mathrm{N}_{\omega}^{\varphi \psi}(\lambda)$ for the pair $\{\varphi, \psi\}$ takes the form

$$
\mathrm{N}_{\omega}^{\varphi \psi}(\lambda)=\frac{\psi(\lambda) \phi(\bar{\omega})-\phi(\lambda) \psi(\bar{\omega})}{\lambda-\bar{\omega}}, \quad \lambda, \omega \in \rho(\widetilde{A}) .
$$

It follows from Definition (2.4) that

$$
\begin{aligned}
& \mathrm{N}_{\omega}^{\varphi \psi}(\lambda)=\frac{\psi(\lambda)-\psi(\omega)^{*}}{\lambda-\bar{\omega}}-\psi(\lambda) \psi(\omega)^{*} \\
&\left.\left.=P_{\mathcal{L}} \frac{R_{\lambda}-R_{\bar{\omega}}}{\lambda-\bar{\omega}}\right\rceil_{\mathcal{L}}-P_{\mathcal{L}} R_{\lambda} P_{\mathcal{L}} R_{\bar{\omega}}\right\rceil_{\mathcal{L}} \\
&\left.\quad=P_{\mathcal{L}} R_{\lambda} P_{\mathcal{H}} R_{\bar{\omega}}\right\rceil_{\mathcal{L}}
\end{aligned}
$$

where $R_{\lambda}=(\widetilde{A}-\lambda)^{-1}$ is a resolvent of lineal relation $\widetilde{A}$. Let $\omega_{j}$ belongs to $\rho(A), u_{j}$ belongs to space $\mathcal{L}$ and $\xi_{j}$ belongs to space $\mathbb{C}^{n}$ for $j=1, \ldots, n$. Then

$$
\begin{gathered}
\sum_{j, k=1}^{n}\left(\mathrm{~N}_{\omega_{j}}^{\varphi \psi}\left(\omega_{k}\right) u_{j}, u_{k}\right)_{\mathcal{L}} \xi_{j} \bar{\xi}_{k}=\sum_{j, k=1}^{n}\left(\left(P_{\mathcal{L}} R_{\omega_{k}} P_{\mathcal{H}} R_{\left.\bar{\omega}_{j}\right|_{\mathcal{L}}}\right) u_{j}, u_{k}\right)_{\mathcal{L}} \xi_{j} \bar{\xi}_{k} \\
=\sum_{j, k=1}^{n}\left[P_{\mathcal{H}} R_{\bar{\omega}_{j}} u_{j}, P_{\mathcal{H}} R_{\bar{\omega}_{k}} u_{k}\right]_{\mathcal{H}} \xi_{j} \bar{\xi}_{k}=\sum_{j, k=1}^{n}\left[g_{j}, g_{k}\right]_{\mathcal{H}} \xi_{j} \bar{\xi}_{k},
\end{gathered}
$$

where $g_{j}=P_{\mathcal{H}} R_{\bar{\omega}_{j}} u_{j}$. Since $\mathcal{H}$ is $\Pi_{k}$-space and $u_{j}(j=1, \ldots, n)$ are arbitrary vectors in $\mathcal{L}$ then the quadratic form (2.8) has $\kappa^{\prime}$ negative squares, where $\kappa^{\prime} \leq \kappa$. Thus property (i) of Definition 1.1 is proved.

The property (ii) is easily checked. Obviously $\varphi(\lambda)-\lambda \psi(\lambda) \equiv I_{\mathcal{L}}$ for all $\lambda \in \rho(\widetilde{A})$ and, hence, the pair $\{\phi, \psi\}$ is a normalized $N_{\kappa^{\prime}}$-pair.

If the relation $\widetilde{A}$ is $\mathcal{L}$-minimal then the set

$$
\operatorname{span}\left\{P_{\mathcal{H}} R_{\omega} u: \omega \in \rho(\widetilde{A}), u \in \mathcal{L}\right\}
$$

is dense in the space $\mathcal{H}$. In this case the quadratic form (2.8) has exactly $\kappa$ negative squares and hence the kernel $\mathrm{N}_{\omega}^{\varphi \psi}(\lambda)$ has $\kappa$ negative squares. Thus the pair $\{\phi, \psi\}$ is a normalized $N_{\kappa}$-pair. 
Definition 2.2. The pair of operator valued functions $\{\varphi, \psi\}$ determined by (2.4) will be called the $N_{\kappa}$-pair corresponding to the selfadjoint linear relation $\widetilde{A}$ and the scale $\mathcal{L}$.

Note that if the vector values functions $\varphi(\lambda)$ and $\psi(\lambda)$ are defined by (2.4) then $\mathfrak{h}_{\varphi \psi}=\mathfrak{h}_{\varphi}=\mathfrak{h}_{\psi}$.

\section{Functional model of a selfadjoint linear relation}

Consider the reproducing kernel Pontryagin space $\mathcal{H}(\phi, \psi)$, which is characterized by the properties:

(1) $\mathrm{N}_{\omega}^{\phi \psi}(\cdot) u \in \mathcal{H}(\phi, \psi)$ for all $\omega \in \mathfrak{h}_{\varphi \psi}$ and $u \in \mathcal{L}$;

(2) for every $f \in \mathcal{H}(\phi, \psi)$ the following identity holds

$$
\left[f(\cdot), \mathrm{N}_{\omega}^{\phi \psi}(\cdot) u\right]_{\mathcal{H}(\phi, \psi)}=(f(\omega), u)_{\mathcal{L}}, \quad \omega \in \mathfrak{h}_{\varphi, \psi}, u \in \mathcal{L} .
$$

It follows from (3.9) that the evaluation operator

$$
E(\lambda): f \mapsto f(\lambda) \quad(f \in \mathcal{H}(\phi, \psi))
$$

is a bounded operator from $\mathcal{H}(\phi, \psi)$ to $\mathcal{L}$. Also note that the set of functions $\left\{\mathrm{N}_{\omega}^{\phi \psi}(\cdot) u: \omega \in \mathfrak{h}_{\varphi \psi}, u \in \mathcal{L}\right\}$ is total in $\mathcal{H}(\varphi, \psi)$ ([2]).

In the next theorem we give functional model of a selfadjoint linear relation $\widetilde{A}$ recovered from a $N_{\kappa}$-pair.

Theorem 3.1. Let $\mathcal{L}$ be a Hilbert space and let $\{\phi, \psi\}$ be a normalized $N_{\kappa}$-pair. Then the linear relation

$$
\begin{aligned}
& A(\phi, \psi)=\left\{\left\{\left[\begin{array}{l}
f \\
u
\end{array}\right],\left[\begin{array}{l}
f^{\prime} \\
u^{\prime}
\end{array}\right]\right\}:\right. \\
& \left.\begin{array}{l}
f, f^{\prime} \in \mathcal{H}(\phi, \psi), u, u^{\prime} \in \mathcal{L}, \\
f^{\prime}(\lambda)-\lambda f(\lambda)=\phi(\lambda) u-\psi(\lambda) u^{\prime}, \lambda \in \mathfrak{h}_{\varphi \psi}
\end{array}\right\}
\end{aligned}
$$

is a selfadjoint linear relation in $\mathcal{H}(\phi, \psi) \oplus \mathcal{L}$ and the normalized pair $\{\phi, \psi\}$ is the $N_{\kappa}$-pair corresponding to $A(\phi, \psi)$ and $\mathcal{L}$.

Proof. Step 1. Let us show that $A(\phi, \psi)$ contains vectors of the form

$$
\left\{F_{\omega} v, F_{\omega}^{\prime} v\right\}:=\left\{\left[\begin{array}{c}
\mathrm{N}_{\omega}(\cdot) v \\
\psi(\bar{\omega}) v
\end{array}\right],\left[\begin{array}{c}
\bar{\omega} \mathrm{N}_{\omega}(\cdot) v \\
\phi(\bar{\omega}) v
\end{array}\right]\right\}, \quad v \in \mathcal{L}, \omega \in \mathfrak{h}_{\varphi \psi},
$$


where $\mathrm{N}_{\omega}(\cdot):=\mathrm{N}_{\omega}^{\phi \psi}(\cdot)$ and

$$
A^{\prime}:=\operatorname{span}\left\{\left\{F_{\omega} v, F_{\omega}^{\prime} v\right\}: v \in \mathcal{L}, \omega \in \mathfrak{h}_{\varphi \psi}\right\}
$$

is a symmetric linear relation.

Indeed, it follows from (3.10) and the equality

$$
(\bar{\omega}-\lambda) \mathrm{N}_{\omega}(\lambda) v=\phi(\bar{\lambda})^{*} \psi(\bar{\omega}) v-\psi(\bar{\lambda})^{*} \phi(\bar{\omega}) v
$$

that $\left\{F_{\omega} v, F_{\omega}^{\prime} v\right\} \in A(\phi, \psi)$.

For arbitrary $\omega_{j} \in \mathfrak{h}_{\varphi \psi}, v_{j} \in \mathcal{L}(j=1,2)$ one obtains

$$
\begin{aligned}
& {\left[\bar{\omega}_{1} \mathrm{~N}_{\omega_{1}}(\cdot) v_{1}, \mathrm{~N}_{\omega_{2}}(\cdot) v_{2}\right]_{\mathcal{H}(\phi, \psi)}-\left[\mathrm{N}_{\omega_{1}}(\cdot) v_{1}, \bar{\omega}_{2} \mathrm{~N}_{\omega_{2}}(\cdot) v_{2}\right]_{\mathcal{H}(\phi, \psi)} } \\
&+\left(\phi\left(\bar{\omega}_{1}\right) v_{1}, \psi\left(\bar{\omega}_{2}\right) v_{2}\right)_{\mathcal{L}}-\left(\psi\left(\bar{\omega}_{1}\right) v_{1}, \phi\left(\bar{\omega}_{2}\right) v_{2}\right)_{\mathcal{L}} \\
&=\left(\bar{\omega}_{1}-\omega_{2}\right)\left(\mathrm{N}_{\omega_{1}}\left(\omega_{2}\right) v_{1}, v_{2}\right)_{\mathcal{L}} \\
&-\left(\left(\phi\left(\bar{\omega}_{2}\right)^{*} \psi\left(\bar{\omega}_{1}\right)-\psi\left(\bar{\omega}_{2}\right)^{*} \phi\left(\bar{\omega}_{1}\right)\right) v_{1}, v_{2}\right)_{\mathcal{L}}=0
\end{aligned}
$$

therefore, $A^{\prime}$ is symmetric in $\mathcal{H}(\phi, \psi) \oplus \mathcal{L}$.

Step 2. Let us show that $\operatorname{ran}\left(A^{\prime}-\lambda\right)$ is dense in $\mathcal{H}(\phi, \psi) \oplus \mathcal{L}$ for $\lambda \in$ $\mathfrak{h}_{\varphi \psi}$. Choose the vector $\left\{F_{\omega} v, F_{\omega}^{\prime} v\right\}$ with $\omega=\bar{\lambda}$. Since $\phi(\lambda)-\lambda \psi(\lambda)=I_{\mathcal{L}}$ then

$$
\begin{array}{r}
\left\{F_{\bar{\lambda}} v, F_{\bar{\lambda}}^{\prime} v-\lambda F_{\bar{\lambda}} v\right\}=\left\{\left[\begin{array}{c}
\mathrm{N}_{\bar{\lambda}}(\cdot) v \\
\psi(\lambda) v
\end{array}\right],\left[\begin{array}{c}
0 \\
\phi(\lambda) v-\lambda \psi(\lambda) v
\end{array}\right]\right\} \\
=\left\{\left[\begin{array}{c}
\mathrm{N}_{\bar{\lambda}}(\cdot) v \\
\psi(\lambda) v
\end{array}\right],\left[\begin{array}{c}
0 \\
v
\end{array}\right]\right\} \in A^{\prime}-\lambda .
\end{array}
$$

Hence $0 \oplus \mathcal{L} \subset \operatorname{ran}\left(A^{\prime}-\lambda\right)$. Taking $\left\{F_{\omega} v, F_{\omega}^{\prime} v\right\}$ with $\omega \neq \bar{\lambda}$ one obtains from (3.11)

$$
\left\{\left[\begin{array}{c}
\mathrm{N}_{\omega}(\cdot) v \\
\psi(\bar{\omega}) v
\end{array}\right],\left[\begin{array}{c}
(\bar{\omega}-\lambda) \mathrm{N}_{\omega}(\cdot) v \\
\phi(\bar{\omega}) v-\lambda \psi(\bar{\omega}) v
\end{array}\right]\right\} \in A^{\prime}-\lambda
$$

and, hence, $\left[\begin{array}{c}\mathrm{N}_{\omega}(\cdot) v \\ 0\end{array}\right] \in \operatorname{ran}\left(A^{\prime}-\lambda\right)$ for all $\omega \neq \bar{\lambda}$. Due to the properties (1) and $(2)$ of $\mathcal{H}(\phi, \psi)$ one obtains the statement. Thus $A^{\prime}$ is an essentially selfadjoint lineal relation and hence $\left(A^{\prime}\right)^{+}$is a selfadjoint lineal relation in $\mathcal{H}(\varphi, \psi) \oplus \mathcal{L}$.

Step 3. Let us show that $A(\phi, \psi)=\left(A^{\prime}\right)^{+}$. Indeed, for every vector

$$
\widehat{F}:=\left\{F, F^{\prime}\right\}=\left\{\left[\begin{array}{c}
f(\cdot) \\
u
\end{array}\right],\left[\begin{array}{c}
f^{\prime}(\cdot) \\
u^{\prime}
\end{array}\right]\right\} \in A(\phi, \psi)
$$


where $f, f^{\prime} \in \mathcal{H}(\phi, \psi)$ and $u, u^{\prime} \in \mathcal{L}$ and arbitrary $\omega \in \mathfrak{h}_{\varphi \phi}, v \in \mathcal{L}$ it follows from (3.10) that

$$
\begin{aligned}
{\left[F^{\prime}, F_{\omega} v\right]_{\mathcal{H}(\phi, \psi) \oplus \mathcal{L}}-} & {\left[F, F_{\omega}^{\prime} v\right]_{\mathcal{H}(\phi, \psi) \oplus \mathcal{L}} } \\
=\left[f^{\prime},\right. & \left.\mathrm{N}_{\omega}(\cdot) v\right]_{\mathcal{H}(\phi, \psi)}-\left[f, \bar{\omega} \mathrm{N}_{\omega}(\cdot) v\right]_{\mathcal{H}(\phi, \psi)} \\
+ & \left(u^{\prime}, \psi(\bar{\omega}) v\right)_{\mathcal{L}}-(u, \phi(\bar{\omega}) v)_{\mathcal{L}} \\
& =\left(f^{\prime}(\omega)-\omega f(\omega)+\psi(\bar{\omega})^{*} u^{\prime}-\phi(\bar{\omega})^{*} u, v\right)_{\mathcal{L}}=0 .
\end{aligned}
$$

Hence $\widehat{F} \in\left(A^{\prime}\right)^{+}$and $A(\phi, \psi) \subset\left(A^{\prime}\right)^{+}$. Conversely, if

$$
\left[f^{\prime}, \mathrm{N}_{\omega}(\cdot) v\right]_{\mathcal{H}(\phi, \psi)}-\left[f, \bar{\omega} \mathrm{N}_{\omega}(\cdot) v\right]_{\mathcal{H}(\phi, \psi)}+\left(u^{\prime}, \psi(\bar{\omega}) v\right)_{\mathcal{L}}-(u, \phi(\bar{\omega}) v)_{\mathcal{L}}=0
$$

for some $f, f^{\prime} \in \mathcal{H}(\varphi, \psi), u, u^{\prime} \in \mathcal{L}$ and all $\omega \in \mathfrak{h}_{\varphi, \psi}, v \in \mathcal{L}$, then

$$
f^{\prime}(\omega)-\omega f(\omega)-\left(\phi(\omega) u-\psi(\omega) u^{\prime}\right)=0
$$

and, hence, $\widehat{F} \in A(\phi, \psi)$. This proves that $\left(A^{\prime}\right)^{+} \subset A(\phi, \psi)$, and, hence, $\left(A^{\prime}\right)^{+}=A(\phi, \psi)$. Therefore, $A(\phi, \psi)$ is a selfadjoint lineal relation.

Step 4. Finally, we show that $\{\varphi, \psi\}$ is a pair corresponding to the selfadjoint linear relation $\widetilde{A}$ and the scale $\mathcal{L}$. Indeed, it follows from (3.12) and Definition $1.2\left(\right.$ (iii') that $^{\prime}$

$$
\begin{gathered}
P_{\mathcal{L}}(\widetilde{A}(\phi, \psi)-\lambda)^{-1}\left\lceil_{\mathcal{L}}=\psi(\lambda),\right. \\
I_{\mathcal{L}}+\lambda P_{\mathcal{L}}(\widetilde{A}(\phi, \psi)-\lambda)^{-1}\left\lceil_{\mathcal{L}}=\varphi(\lambda) .\right.
\end{gathered}
$$

Therefore, the pair $\{\varphi, \psi\}$ is a normalized $N_{\kappa}$-pair corresponding to the linear relation $A(\phi, \psi)$ and the scale $\mathcal{L}$.

Remark 3.1. It follows from (3.12) that the linear relation $A(\varphi, \psi)$ given by $(3.10)$ is $\mathcal{L}$-minimal.

Remark 3.2. For every normalized $N_{\kappa}$ pair $\{\varphi, \psi\}$ and $h \in \mathcal{H}(\varphi, \psi)$ the following identity holds

$$
P_{\mathcal{L}}(A(\varphi, \psi)-\lambda)^{-1}\left[\begin{array}{l}
h \\
0
\end{array}\right]=h(\lambda), \quad\left(\lambda \in \mathfrak{h}_{\varphi \psi}\right) .
$$

Indeed, it follows from (3.12) that for every $v \in \mathcal{L}$ one obtains

$$
\begin{aligned}
\left(P_{\mathcal{L}}(A(\varphi, \psi)\right. & \left.-\lambda)^{-1}\left[\begin{array}{l}
h \\
0
\end{array}\right], v\right)_{\mathcal{L}} \\
& =\left[\left[\begin{array}{l}
h \\
0
\end{array}\right],(A(\varphi, \psi)-\bar{\lambda})^{-1}\left[\begin{array}{l}
0 \\
v
\end{array}\right]_{\mathcal{H}(\varphi, \psi) \oplus \mathcal{L}}\right.
\end{aligned}
$$




$$
=\left[h, \mathrm{~N}_{\lambda}(\cdot) v\right]_{\mathcal{H}(\varphi, \psi)}=(h(\lambda), v)_{\mathcal{L}} .
$$

Therefore, $E(\lambda)=P_{\mathcal{L}}(A(\varphi, \psi)-\lambda)^{-1}\left\lceil_{\mathcal{H}(\varphi, \psi)}\right.$ is the evaluation operator in $\mathcal{H}(\varphi, \psi)$.

We define the lineal space $\widetilde{\mathfrak{N}}_{\omega}$ via the formula

$$
\widetilde{\mathfrak{N}}_{\omega}:=\left\{\mathrm{N}_{\bar{\omega}}^{\varphi} \psi(\cdot) u, u \in \mathcal{L}\right\} .
$$

Proposition 3.1. Let $\{\varphi, \psi\}$ be a normalized $N_{\kappa}$-pair in the space $\mathcal{L}$. Then

(i) the space $\widetilde{\mathfrak{N}}_{\bar{\omega}}$ is a positive subspace in $\mathcal{H}(\varphi, \psi)$ if and only if $\mathrm{N}_{\omega}^{\varphi \psi}(\omega)$ is a strictly positive operator in $\mathcal{L}$.

(ii) if additionally $\bigcap_{\lambda} \operatorname{ker} \aleph_{\omega}^{\varphi \psi}(\lambda)=\{0\}$ then the space $\widetilde{\mathfrak{N}}_{\bar{\omega}}$ is a degenerate subspace in $\mathcal{H}(\varphi, \psi)$ if and only if 0 is an eigenvalue of $\mathrm{N}_{\omega}^{\varphi \psi}(\omega)$.

Proof. Denote $\mathrm{N}_{\omega}(\cdot):=\mathrm{N}_{\omega}^{\varphi \psi}(\cdot)$. Let us prove the first statement. Since

$$
\left[\mathrm{N}_{\omega}(\cdot) u, \mathrm{~N}_{\omega}(\cdot) u\right]_{\mathcal{H}(\varphi, \psi)}=\left(\mathrm{N}_{\omega}(\omega) u, u\right)_{\mathcal{L}} \quad(u \in \mathcal{L})
$$

then a conditions $\mathrm{N}_{\omega}(\omega)>0$ is equivalent to the inequality $\left(\mathrm{N}_{\omega}(\cdot) u, \mathrm{~N}_{\omega}(\cdot) u\right)_{\mathcal{H}(\varphi, \psi)}>0$ which holds for all $(0 \neq) u \in \mathcal{L}$.

Now we prove the second statement. Let at first the space $\widetilde{\mathfrak{N}}_{\bar{\omega}}$ is a degenerate subspace. Then exist $(0 \neq) u_{0} \in \mathcal{L}$ such that

$$
0=\left[\mathrm{N}_{\omega}(\cdot) u_{0}, \mathrm{~N}_{\omega}(\cdot) v\right]_{\mathcal{H}(\varphi, \psi)}=\left(\mathrm{N}_{\omega}(\omega) u_{0}, v\right)_{\mathcal{L}}
$$

which holds for all $v \in \mathcal{L}$. Therefore $\mathrm{N}_{\omega}(\omega) u_{0}=0$ and hence 0 is an eigenvalue of $\mathrm{N}_{\omega}(\omega)$.

Conversely, let $\mathrm{N}_{\omega}(\omega) u_{0}=0$ where $(0 \neq) u_{0} \in \mathcal{L}$. Then

$$
0=\left(\mathrm{N}_{\omega}(\omega) u_{0}, v\right)_{\mathcal{L}}=\left[\mathrm{N}_{\omega}(\cdot) u_{0}, \mathrm{~N}_{\omega}(\cdot) v\right]_{\mathcal{H}(\varphi, \psi)},
$$

therefore $\mathbf{N}_{\omega}(\cdot) u_{0}$ is orthogonal to the space $\widetilde{\mathfrak{N}}_{\omega}$. Since $\mathbf{N}_{\omega}(\cdot) u_{0} \not \equiv 0$ then it is a nontrivial isotropic vector in the space $\widetilde{\mathfrak{N}}_{\omega}$.

Proposition 3.2. Let $\widetilde{A}$ be a selfadjoint linear relation in $\mathcal{H} \oplus \mathcal{L}$ and let $\{\varphi, \psi\}$ be the normalized Nevanlinna pair given by (2.4). Let the operator valued function $\gamma(\lambda): \mathcal{L} \rightarrow \mathcal{H}$ be defined by

$$
\gamma(\lambda):=\left.P_{\mathcal{H}}(\widetilde{A}-\lambda)^{-1}\right|_{\mathcal{L}} \quad(\lambda \in \rho(\widetilde{A})) .
$$

Then the following identity holds

$$
\mathrm{N}_{\omega}^{\varphi \psi}(\lambda)=\gamma(\bar{\lambda})^{*} \gamma(\bar{\omega}) \text {. }
$$


Proof. Indeed, it follows from (2.7) that the kernel $\mathrm{N}_{\omega}^{\varphi \psi}(\lambda)$ takes the form

$$
\mathrm{N}_{\omega}^{\varphi \psi}(\lambda)=\left(P_{\mathcal{L}} R_{\lambda} P_{\mathcal{H}}\right)\left(\left.P_{\mathcal{H}} R_{\bar{\omega}}\right|_{\mathcal{L}}\right)=\gamma(\bar{\lambda})^{*} \gamma(\bar{\omega}) .
$$

Proposition 3.3. Let $\{\varphi, \psi\}$ be a normalized $N_{\kappa}$-pair in the space $\mathcal{L}$. Then $\widetilde{\mathfrak{N}}_{\omega}$ is a closed space if and only if $\mathrm{N}_{\omega}(\omega)$ is normally solvable.

Proof. Denote $B:=\mathrm{N}_{\omega}^{\varphi \psi}(\omega)$ and consider its spectral decomposition

$$
B=B_{+} \oplus B_{-} \oplus B_{0}
$$

and the corresponding decomposition of the Hilbert space $\mathcal{L}$

$$
\mathcal{L}=\mathcal{L}_{+} \oplus \mathcal{L}_{-} \oplus \mathcal{L}_{0}
$$

where $B_{+}>0, B_{-}<0$, and $B_{0}=0_{\mathcal{L}_{0}}$. It follows from (3.12) and (3.15) that

$$
\gamma(\omega) v=\mathrm{N}_{\bar{\omega}}^{\varphi \psi}(\cdot) v \quad \forall v \in \mathcal{L} .
$$

Since $\widetilde{\mathfrak{N}}_{\omega}=\gamma(\omega) \mathcal{L}$ then $\widetilde{\mathfrak{N}}_{\omega}$ can be decomposed as

$$
\widetilde{\mathfrak{N}}_{\omega}=\mathfrak{N}_{\omega}^{+}[+] \mathfrak{N}_{\omega}^{-}[+] \mathfrak{N}_{\omega}^{0}
$$

where $\mathfrak{N}_{\omega}^{ \pm}=\gamma(\omega) \mathcal{L}_{ \pm}$and $\mathfrak{N}_{\omega}^{0}=\gamma(\omega) \mathcal{L}_{0}$. This decomposition is orthogonal since $\mathrm{N}_{\omega}(\omega)=\mathrm{N}_{\bar{\omega}}(\bar{\omega})$. For instance, if $v_{+} \in \mathcal{L}_{+}, v_{-} \in \mathcal{L}_{-}$then

$$
\begin{aligned}
{\left[\gamma(\omega) v_{+}, \gamma(\omega) v_{-}\right]_{\mathcal{H}(\varphi, \psi)} } & =\left[\mathrm{N}_{\bar{\omega}}(\cdot) v_{+}, \mathrm{N}_{\bar{\omega}}(\cdot) v_{-}\right]_{\mathcal{H}(\varphi, \psi)} \\
& =\left(\mathrm{N}_{\bar{\omega}}(\bar{\omega}) v_{+}, v_{-}\right)_{\mathcal{L}}=\left(\mathrm{N}_{\omega}(\omega) v_{+}, v_{-}\right)_{\mathcal{L}}=0 .
\end{aligned}
$$

Let $B=\mathrm{N}_{\omega}^{\varphi \psi}(\omega)$ be normally solvable $\operatorname{ran} B$ is closed in $\mathcal{L}$. Since $\mathcal{L}_{-}$ and $\mathcal{L}_{0}$ are finite-dimensional subspaces in $\mathcal{L}$ then $\operatorname{ran} B_{+}$is closed and by Banach Theorem there is $c>0$ such that

$$
\left(B_{+} v, v\right)_{\mathcal{L}} \geq c^{2}\|v\|_{\mathcal{L}}^{2} \quad\left(v \in \mathcal{L}^{+}\right) .
$$

Due to Proposition 3.2 it can be rewritten as

$$
[\gamma(\omega) v, \gamma(\omega) v]_{\mathcal{H}(\varphi, \psi)} \geq c^{2}\|v\|_{\mathcal{L}}^{2} \quad\left(v \in \mathcal{L}^{+}\right) .
$$

Thus $\mathfrak{N}_{\omega}^{+}=\gamma_{(\omega)}(\omega)$ is closed. Since $\operatorname{dim} \mathfrak{N}_{\omega}^{-} \leq \kappa$ and $\operatorname{dim} \mathfrak{N}_{\omega}^{0} \leq \kappa$ this implies that $\widetilde{\mathfrak{N}}_{\omega}$ is closed.

Conversely, if $\widetilde{\mathfrak{N}}_{\omega}$ is closed, then $\mathfrak{N}_{\omega}^{+}$is also closed. Since $\gamma(\omega) \curlyvee_{\mathcal{L}_{+}}$ is invertible, then there is $c>0$ such that (3.21) holds. In view of Proposition 3.2 this implies that $\operatorname{ran} B_{+}$is closed in $\mathcal{L}$. Since $B_{-}$is finite-dimensional then $\operatorname{ran} B$ is closed. This proves the statement. 
Remark 3.3. If $m$ is a function from the class $N_{\kappa}(\mathcal{L})$ such that $I-\lambda m(\lambda)$ is invertible for all $\lambda \in \mathbb{C} \backslash \mathbb{R}$ then the pair $\left\{I_{\mathcal{L}}, m(\lambda)\right\}$ is equivalent to the normalized $N_{\kappa}$-pair

$$
\{\varphi, \psi\}=\left\{\left(I_{\mathcal{L}}-\lambda m(\lambda)\right)^{-1}, m(\lambda)\left(I_{\mathcal{L}}-\lambda m(\lambda)\right)^{-1}\right\}
$$

and the corresponding model operator can be rewritten as

$$
A(\phi, \psi)=\left\{\left\{\left[\begin{array}{l}
f \\
u
\end{array}\right],\left[\begin{array}{l}
f^{\prime} \\
u^{\prime}
\end{array}\right]\right\}: \begin{array}{l}
f, f^{\prime} \in \mathcal{H}(\phi, \psi), \quad u, u^{\prime} \in \mathcal{L} ; \\
f^{\prime}(\lambda)-\lambda f(\lambda)=u-m(\lambda) u^{\prime}, \lambda \in \mathbb{C} \backslash \mathbb{R}
\end{array}\right\} .
$$

Considering the projection of this model to the space $\mathcal{H}(\varphi, \psi)$, one obtains the model for a symmetric operator $S$ with the abstract Weyl function $m(\lambda)$, given in [10] in the Hilbert space case and in [8] in the Pontryagin space case. In particular, a model for a selfadjoint extension $A_{0}$ of $S$ can be derived from (3.22) in the form

$$
A_{0}=\left\{\left\{f, f^{\prime}\right\} \in \mathcal{H}(\phi, \psi)^{2}: f^{\prime}(\lambda)-\lambda f(\lambda) \equiv u \text { for some } u \in \mathcal{L}\right\} .
$$

This reproducing kernel space model appeared originally in [1].

\section{Generalized Fourier transform}

In this section we show that every $\mathcal{L}$-minimal selfadjoint linear relation $A$ is unitarily equivalent to its functional model $A(\varphi, \psi)$, constructed in Theorem 3.1. The operator $\mathcal{F}: \mathcal{H} \rightarrow \mathcal{H}(\varphi, \psi)$ given by the formula

$$
h \mapsto(\mathcal{F} h)(\lambda)=\gamma(\bar{\lambda})^{*} h=P_{\mathcal{L}}(\widetilde{A}-\lambda)^{-1} h \quad(h \in \mathcal{H})
$$

is called the generalized Fourier transform associated with $\widetilde{A}$ and the scale $\mathcal{L}$.

Theorem 4.1. Let $\widetilde{A}$ be a selfadjoint linear relation in $\mathcal{H} \oplus \mathcal{L}$ and let $\{\varphi, \psi\}$ be the corresponding $N_{\kappa}$-pair given by (2.4). Then:

1) The generalized Fourier transform $\mathcal{F}$ maps isometrically the subspace $\mathcal{H}_{0}$ onto $\mathcal{H}(\varphi, \psi)$ and $\mathcal{F}$ is identically equal to 0 on $\mathcal{H} \ominus \mathcal{H}_{0}$;

2) For every $\left\{\left[\begin{array}{c}f \\ u\end{array}\right],\left[\begin{array}{c}f^{\prime} \\ u^{\prime}\end{array}\right]\right\} \in \widetilde{A}$ the following identity holds

$$
E(\lambda) \mathcal{F}\left(f^{\prime}-\lambda f\right)=[\varphi(\lambda)-\psi(\lambda)]\left[\begin{array}{c}
u \\
u^{\prime}
\end{array}\right]
$$


Proof. 1) For every vector $h=\gamma(\bar{\omega}) v(\omega \in \rho(\widetilde{A}), v \in \mathcal{L})$ it follows from Proposition 3.2 that

$$
(\mathcal{F} h)(\lambda)=\gamma(\bar{\lambda})^{*} \gamma(\bar{\omega}) v=\mathbf{N}_{\omega}^{\varphi \psi}(\lambda) v
$$

Therefore, $\mathcal{F}$ maps the linear space $\operatorname{span}\{\gamma(\bar{\omega}) \mathcal{L}: \omega \in \rho(\widetilde{A})\}$ which is dense in $\mathcal{H}_{0}$ onto the linear space $\operatorname{span}\left\{\mathrm{N}_{\omega}^{\varphi \psi}(\cdot) \mathcal{L}: \omega \in \rho(\widetilde{A})\right\}$ which is dense in $\mathcal{H}(\varphi, \psi)$. Moreover, this mapping is isometric, since

$$
\begin{aligned}
{[\mathcal{F} h, \mathcal{F} h]_{\mathcal{H}(\varphi, \psi)}=\left[\mathrm{N}_{\omega}^{\varphi \psi}(\cdot) v, \mathbf{N}_{\omega}^{\varphi \psi}(\cdot) v\right]_{\mathcal{H}(\varphi, \psi)} } \\
=\left(\mathbf{N}_{\omega}^{\varphi \psi}(\omega) v, v\right)_{\mathcal{L}}=[h, h]_{\mathcal{H}} .
\end{aligned}
$$

This proves the first statement. It is clear from (4.24) that $\mathcal{F} h \equiv 0$ for $h \in \mathcal{H} \ominus \mathcal{H}_{0}$.

2) Let $h=\gamma(\bar{\omega}) v=P_{\mathcal{H}}(\widetilde{A}-\bar{\omega})^{-1} v, v \in \mathcal{L}$. It follows from $(2.4),(3.15)$ that

$$
\left[\begin{array}{c}
h \\
\psi(\bar{\omega}) v
\end{array}\right]=(\widetilde{A}-\bar{\omega})^{-1}\left[\begin{array}{l}
0 \\
v
\end{array}\right], \quad\left[\begin{array}{c}
\bar{\omega} h \\
\varphi(\bar{\omega}) v
\end{array}\right]=\left(I+\bar{\omega}(\widetilde{A}-\bar{\omega})^{-1}\right)\left[\begin{array}{l}
0 \\
v
\end{array}\right]
$$

and hence

$$
\left\{\left[\begin{array}{c}
h \\
\psi(\bar{\omega}) v
\end{array}\right],\left[\begin{array}{c}
\bar{\omega} h \\
\varphi(\bar{\omega}) v
\end{array}\right]\right\} \in \widetilde{A}
$$

Since $\widetilde{A}=\widetilde{A}^{*}$ one obtains for all $\left\{\left[\begin{array}{l}f \\ u\end{array}\right],\left[\begin{array}{l}f^{\prime} \\ u^{\prime}\end{array}\right]\right\} \in \widetilde{A}$

$$
\left[f^{\prime}, h\right]_{\mathcal{H}}-[f, \bar{\omega} h]_{\mathcal{H}}+\left(u^{\prime}, \psi(\bar{\omega})\right)_{\mathcal{L}}-(u, \varphi(\bar{\omega}) v)_{\mathcal{L}}=0, \quad v \in \mathcal{L} .
$$

This implies

$$
\gamma(\bar{\omega})^{*}\left(f^{\prime}-\bar{\omega} f\right)=\varphi(\omega) u-\psi(\omega) u^{\prime}, \quad \omega \in \rho(\widetilde{A}) .
$$

This proves the identity (4.25).

Corollary 4.1. In the case, when the linear relation $\widetilde{A}$ is $\mathcal{L}$-minimal it is unitary equivalent to the linear relation $A(\varphi, \psi)$ via the formula

$$
A(\varphi, \psi)=\left\{\left\{\left[\begin{array}{c}
\mathcal{F} f \\
u
\end{array}\right],\left[\begin{array}{c}
\mathcal{F} f^{\prime} \\
u^{\prime}
\end{array}\right]\right\}:\left\{\left[\begin{array}{l}
f \\
u
\end{array}\right],\left[\begin{array}{l}
f^{\prime} \\
u^{\prime}
\end{array}\right]\right\} \in \widetilde{A}\right\} .
$$

The operator $\mathcal{F} \oplus I_{\mathcal{L}}$ establishes this unitary equivalence.

Corollary 4.2. It follows from (3.13) that the Fourier transform $\mathcal{F}$ associated with the operator $A(\varphi, \psi)$ is identical, since

$$
(\mathcal{F} h)(\lambda)=P_{\mathcal{L}}(A(\varphi, \psi)-\lambda)^{-1}\left[\begin{array}{l}
h \\
0
\end{array}\right]=h(\lambda) \quad \text { for every } h \in \mathcal{H}(\varphi, \psi) .
$$


Lemma 4.1. Let $\widetilde{A}$ be a selfadjoint linear relation in $\mathcal{H} \oplus \mathcal{L}$, let $\{\varphi, \psi\}$ be the normalized $N_{\kappa}$-pair given by (2.4). Then the following implications hold

(i) $\operatorname{ker} \psi(\lambda)=\{0\}$ for some $\lambda \in \rho(\widetilde{A}) \Rightarrow P_{\mathcal{L}} \operatorname{dom} \widetilde{A}$ is dense in $\mathcal{L}$;

(ii) $\operatorname{ker} \varphi(\lambda)=\{0\}$ for some $\lambda \in \rho(\widetilde{A}) \Rightarrow P_{\mathcal{L}} \operatorname{ran} \widetilde{A}$ is dense in $\mathcal{L}$.

If, in addition, the relation $\widetilde{A}$ is $\mathcal{L}$-minimal, and $\mathrm{N}_{\omega}^{\varphi \psi}(\omega)>0$ for some $\omega \in \rho(\widetilde{A})$ then

$$
\operatorname{ker} \varphi(\omega)=\{0\}, \quad \operatorname{ker} \psi(\omega)=\{0\} .
$$

Proof. Let us prove the first statement. The set $P_{\mathcal{L}} \operatorname{dom} \widetilde{A}$ consists of the vectors $u \in \mathcal{L}$ such that

$$
\left\{\left[\begin{array}{l}
f \\
u
\end{array}\right],\left[\begin{array}{c}
f^{\prime} \\
u^{\prime}
\end{array}\right]\right\} \in \widetilde{A} \quad \text { for some } \quad f, f^{\prime} \in \mathcal{H}, u^{\prime} \in \mathcal{L}
$$

If there is a vector $v \in \mathcal{L}$ such that $v \perp u$ for all $u \in P_{\mathcal{L}} \operatorname{dom} \widetilde{A}$ then

$$
\left\{\left[\begin{array}{l}
0 \\
0
\end{array}\right],\left[\begin{array}{l}
0 \\
v
\end{array}\right]\right\} \in \widetilde{A}
$$

and then $\psi(\lambda) v=0$, due to (2.4). But $\operatorname{ker} \psi(\lambda)=\{0\}$ therefore $v=0$.

The proof of the second statement is similar.

Assume now that $\mathrm{N}_{\omega}^{\varphi \psi}(\omega)>0$ for some $\omega \in \rho(\widetilde{A})$ and that $\psi(\omega) v=0$. Then in view of $(2.4) \varphi(\omega) v=v$ and

$$
\begin{aligned}
\left(\mathrm{N}_{\omega}(\omega) v, v\right)_{\mathcal{L}} & =\left(\mathrm{N}_{\bar{\omega}}(\bar{\omega}) v, v\right)_{\mathcal{L}} \\
& =\frac{1}{\omega-\bar{\omega}}((\psi(\bar{\omega}) \varphi(\omega)-\varphi(\bar{\omega}) \psi(\omega)) v, v)_{\mathcal{L}} \\
& =\frac{1}{\omega-\bar{\omega}}(v, \varphi(\omega) v)_{\mathcal{L}}=0 .
\end{aligned}
$$

This implies $v=0$.

Criterions for the right parts in (i) and (ii) to be true are given in the following lemma.

Lemma 4.2. Let $\widetilde{A}$ be a $\mathcal{L}$-minimal selfadjoint linear relation in $\mathcal{H} \oplus \mathcal{L}$, let $\{\varphi, \psi\}$ be the normalized $N_{\kappa}$-pair given by (2.4). Then

(i) $\bigcap_{\lambda \in \rho(\widetilde{A})} \operatorname{ker} \psi(\lambda)=\{0\}$ if and only if $P_{\mathcal{L}} \operatorname{ran} \widetilde{A}$ is dense in $\mathcal{L}$;

(ii) $\bigcap_{\lambda \in \rho(\widetilde{A})} \operatorname{ker} \varphi(\lambda)=\{0\}$ if and only if $P_{\mathcal{L}} \operatorname{ran} \widetilde{A}$ is dense in $\mathcal{L}$. 
Proof. The necessity of (i) and (ii) follows from Lemma 4.1. To prove the sufficiency let us consider the linear relation $A(\varphi, \psi)$ given by (3.10). By Corollary $4.1 A(\varphi, \psi)$ is unitary equivalent to the linear relation $\widetilde{A}$ and, hence, we may prove the statement for the linear relation $A(\varphi, \psi)$.

Assume that $\psi(\lambda) v=0$ for some $v \in \mathcal{L}$ and for all $\lambda \in \rho(A(\varphi, \psi))$. Then in view of $(2.4) \varphi(\lambda) v=v$ and

$$
\mathrm{N}_{\bar{\lambda}}^{\varphi \psi}(\omega) v=\frac{1}{\omega-\lambda}(\psi(\omega) \varphi(\lambda)-\varphi(\omega) \psi(\lambda)) v=0
$$

for all $\lambda, \omega \in \rho(A(\varphi, \psi))$. Now it follows from (3.11) that

$$
\left\{\left[\begin{array}{l}
0 \\
0
\end{array}\right],\left[\begin{array}{l}
0 \\
v
\end{array}\right]\right\}=\left\{\left[\begin{array}{c}
\mathrm{N}_{\omega}^{\varphi \psi}(\cdot) v \\
0
\end{array}\right],\left[\begin{array}{c}
\bar{\omega} \mathrm{N}_{\omega}^{\varphi \psi}(\cdot) v \\
v
\end{array}\right]\right\} \in A(\varphi, \psi) .
$$

and, hence, $v \perp P_{\mathcal{L}} \operatorname{dom} \widetilde{A}$.

Acknowledgments. I express my gratitude to V. A. Derkach for guidance in the work and many useful discussions. I am also grateful to M. M. Malamud for valuable remarks and suggestions.

\section{References}

[1] D. Alpay, P. Bruinsma, A. Dijksma, H. S. V. de Snoo, A Hilbert space associated with a Nevanlinna function // Proceeding MTNS meeting Amsterdam, (1989), $115-122$.

[2] D. Alpay, A. Dijksma, J. Rovnyak, H. S. V. de Snoo, Schur functions, operator colligations, and reproducing kernel Pontryagin spaces/ Oper. Theory: Adv. Appl., 96, Birkhäuser Verlag, Basel, 1997.

[3] T. Ya. Azizov and I. S. Iohvidov, Linear operators in spaces with indefinite metric, Nauka, Moscow, 1986 (Russian) [English translation: John Wiley, New York, 1989].

[4] J. Behrndt, S. Hassi, and H. S. V. de Snoo, Functional models for Nevanlinna families // Opuscula Math., 28 (2008), 233-245.

[5] J. Behrndt, S. Hassi, and H. S. V. de Snoo, Boundary relations, unitary colligations, and functional models // Complex Anal. Oper. Theory, 3 (2009), 57-98.

[6] V. A. Derkach, Abstract interpolation problem in Nevanlinna classes // Oper. Theory Adv. Appl., 190 (2009), 197-236.

[7] V. A. Derkach, S. Hassi, M. M. Malamud, and H. S. V. de Snoo, Boundary relations and their Weyl families // Trans. Amer. Math. Soc., 358 (2006), 53515400.

[8] V. A. Derkach, S. Hassi, H. S. V. de Snoo, Operator models associated with Kac subclasses of genaralized Nevanlinna functions // Methods of Functional Analysis and Topology, 5 (1999), 65-87.

[9] V. A. Derkach and M. M. Malamud, Generalized resolvents and the boundary value problems for hermitian operators with gaps // J. Functional Analysis, 95 (1991), 1-95. 
[10] V. A. Derkach and M. M. Malamud, The extension theory of hermitian operators and the moment problem // J. Math. Sciences, 73 (1995), 141-242.

[11] A. Dijksma and H. S. V. de Snoo, Symmetric and selfadjoint relations in Krein Spaces I // Oper. Theory Adv. Appl., 24 (1987), 145-166.

[12] M. G. Krein, On resolvents of Hermitian operators with defect indices $(m, m) / /$ Dokl. Akad. Nauk SSSR, 52 (1946), N 8, 657-660.

[13] M. G. Krein, G. K. Langer, Defect subspaces and generalized resolvents of an Hermitian operator in the space $\Pi_{\kappa} / /$ Functional Analysis and Its Applications, 5 (1971), N 2, 136-146; 5 (1971), N 3, 54-69.

[14] M. G. Kreı̆n and H. Langer, Über die Q-functions eines $\pi$-hermiteschen Operators im Raume $\Pi_{\kappa} / /$ Acta Sci. Math. (Szeged), 34 (1973), 191-230.

[15] H. Langer, B. Textorius, On generalized resolvents and $Q$-functions of symmetric linear relations in Hilbert spaces // Pacif. J. Math. 72 (1977), N 1, 135-165.

[16] M. M. Malamud, S. M. Malamud, Spectral theory of operator measures in Hilbert space // St.-Petersburg Math. Journal, 15 (2003), N 3, 1-77.

\title{
CONTACT INFORMATION
}

\section{Evgen Neiman}

\author{
Department of Mathematics \\ Donetsk National University \\ Universitetskaya str. 24 \\ 83055 Donetsk \\ Ukraine \\ E-Mail: evg_sqrt@mail.ru
}

